\title{
El Pensamiento Computacional en la Resolución de Problemas Matemáticos en Básica Primaria a través de Computación Desconectada
}

\author{
Floralba Isabel Silva-Calpa ${ }^{1,2}$, Eduardo E. Tonguino ${ }^{1}$, Rafael R. Mantilla ${ }^{1}$ \\ ${ }^{1}$ Facultad de Educación - Universidad de Santander (UDES) -Bucaramanga - \\ (Santander) Colombia \\ ${ }^{2}$ Institución Educativa Municipal Ciudad de Pasto (IEMCP) - Pasto (N) - Colombia \\ floralba.silva@iemciudaddepasto.edu.co, eduquiroz16@gmail.com, \\ rafael.mantilla@cvudes.edu.co
}

\begin{abstract}
Computational Thinking (CT) in mathematics education contributes to students to solve problems with logical thinking and creativity. CT skills can be encouraged not only through computer systems but also with Unplugged Computing activities. In this article, through an Action-Research method, we carry out an empirical study with elementary school students, aiming to propose a Test for Unplugged Computing, which allows diagnosing the level of development in the CT components to solving mathematical problems. This diagnosing enables to create the relevant activities for the students and, therefore, encourages CT.
\end{abstract}

Resumen. E1 Pensamiento Computacional (CT, por sus siglas en inglés) en la enseñanza de las matemáticas contribuye para que los estudiantes resuelvan problemas de forma lógica, reflexiva y creativa. Las habilidades del CT pueden fomentarse no únicamente a través de sistemas computacionales, sino también con actividades de Computación Desconectada. En este artículo, mediante una Investigación-Acción, realizamos un estudio empírico con estudiantes de básica primaria, con miras a proponer un Test para computación desconectada, que permita diagnosticar el nivel de desarrollo en los componentes del CT para la resolución de problemas matemáticos. El diagnóstico permite la creación de actividades adecuadas para los estudiantes $\mathrm{y}$, por lo tanto, para fomentar el CT.

\section{Introducción}

Los avances tecnológicos que se presentan a gran velocidad exigen de la sociedad una preparación acorde para adquirir las habilidades necesarias y hacer frente a los retos del mundo actual en innovación, creación y emprendimiento, siendo necesario que desde la educación primaria se promueva el desarrollo del Pensamiento Computacional (CT, por sus siglas en inglés).

El CT es la habilidad para resolver problemas teniendo en cuenta los conceptos informáticos y la programación, y es fundamental que se enseñe desde la educación primaria [Pérez et al., 2019]. Para la resolución de problemas matemáticos, se debería incluir el CT considerando la capacidad analítica del niño, esto permitirá que desarrollen habilidades en los componentes del CT: descomposición, abstracción- 
generalización, reconocimiento de patrones, diseño de algoritmo y paralelización [Wing, 2008]. Descomponer es dividir un problema en partes más pequeñas; abstraergeneralizar, es obtener del problema los datos importantes; reconocimiento de patrones, es determinar similitudes entre los componentes de un problema matemático; diseño de algoritmos, son los pasos que se debe seguir para la resolución de un problema y la paralelización, es la capacidad de realizar actividades de manera simultánea.

Wing (2008), afirma que el CT no es sinónimo de capacidad para programar un ordenador, puesto que requiere pensar en diferentes niveles de abstracción y es independiente de los dispositivos. Por lo tanto, se puede desarrollar CT con actividades sin utilizar ordenador (basta papel y lápiz), lo que se conoce como Computación Desconectada [Bell, et al., 2009]. El CT es una competencia básica para desenvolverse en la sociedad digital, pero no es una habilidad «rutinaria»o «mecánica», ya que es una forma de resolver problemas de manera inteligente e imaginativa, cualidades que no poseen los ordenadores [Berrocoso, et al., 2015].

En la literatura, encontramos diversos estudios sobre la aplicación del CT a la educación básica, entre ellos Yadav, et al., (2016), Rodríguez, (2017), y Ortega, et al., (2016), quienes hablan de la incorporación de los componentes del CT desde temprana edad, algunos proponen el desarrollo de estas habilidades con ordenadores y otros sin ellos puesto que las operaciones mentales de orden superior como analizar, abstraer y descomponer son requeridas para ser competente en una sociedad globalizada.

Diversos autores como Rodríguez, (2017), Shute, et al., (2017) y Doleck, et al., (2017), usan el Test de Pensamiento Computacional, propuesto por Román-Gonzáles, et al., (2015), que consiste en evaluar los componentes de CT en la resolución de problemas desde la programación. Otros estudios como Brennan \& Resnick, (2012), Weintrop, et al., (2015) y de la Fuente y García, (2017), también evalúan el desarrollo del CT a través del uso de la programación con Scratch, un lenguaje de programación visual. Si bien hay estudios enfocados a la Computación Desconectada, hay carencia de ellos sobre la aplicación de un Test para medir los niveles del CT, específicamente para la resolución de problemas matemáticos en básica primaria. Esto motivó el desarrollo de nuestro estudio, el cual, siguiendo un método de Investigación-Acción, tiene como objetivo diagnosticar el nivel de desarrollo en los componentes del CT desde las asignaturas STEAM en la resolución de problemas matemáticos en la básica primaria, con la finalidad de construir un Test de CT orientado a la computación desconectada.

Para contribuir al desarrollo del CT desde la básica primaria implementamos la metodología STEAM, que es un acrónimo de las asignaturas en ingles Science, Technoly, Engineering, Arts, and mathematics. Esta metodología permite a los estudiantes mejorar en la resolución de problemas matemáticos desde ciencias naturales y artística teniendo en cuenta los componentes del CT. Dos Santos, et al. (2019), indican que, en este contexto, es posible realizar un trabajo multidisciplinar, pudiendo integrar Artes, y las otras áreas de la nomenclatura STEAM.

El estudio aquí presentado hace parte un estudio mayor que tiene la finalidad de construir un Test específico para evaluar los componentes del CT para la resolución de problemas matemáticos en básica primaria. En este artículo, presentamos dos ciclos de nuestra Investigación-Acción. En el primer ciclo, identificamos y recolectamos problemas matemáticos de acuerdo al conocimiento de los estudiantes de cuarto grado de básica primaria de la Institución Educativa Municipal Ciudad de Pasto. Con ello, 
tomamos como referencia los componentes del CT propuestos por Wing (2008), para diseñar un Test que permita medir estos componentes desde la resolución de problemas matemáticos con división. Aplicamos el Test a 22 estudiantes y a partir de los resultados, realizamos el segundo ciclo de investigación. En el segundo ciclo, para mejorar la resolución de problemas con los componentes del CT, aplicamos la metodología STEAM a través de un proyecto denominado "Arco Iris" y la interacción con los Recursos Educativos Digitales Abiertos (REDA). El proyecto Arco Iris consiste en la construcción de mini carrozas teniendo en cuenta las figuras geométricas, los ecosistemas y los colores secundarios, los cuales se articulan con la resolución de problemas matemáticos con división, además la interacción con los REDA les permiten desarrollar habilidades en la resolución de problemas. Los resultados encontrados muestran la importancia de hacer un diagnóstico para medir los niveles de desarrollo en los componentes del CT orientado a actividades de computación desconectada. Estos aspectos pueden guiar futuras investigaciones.

\section{Estudios Relacionados}

A continuación, se presentan los estudios relacionados con el contexto de este estudio, tal como evaluación de las habilidades de CT, computación desconectada y el uso de STEAM para desarrollar el CT.

Los componentes de CT, son los pilares del Pensamiento Computacional, siendo la abstracción considerada la esencia de este tipo de pensamiento [Oliveira, et al., 2019]. En el estudio de Pérez, et al., (2019), los autores diagnostican los niveles del CT en estudiantes de educación superior mediante el test de Román-González (2016), a través del lenguaje de programación Scratch y establecen la relación entre las habilidades del CT y la orientación espacial, toma de decisiones, empleo de bucles y elaboración de funciones para la resolución de problemas. Los autores destacan que los estudiantes mostraron altos niveles de dificultad en la toma de decisiones y sentencias de repetición.

De igual forma, Rodríguez (2017), evalúa el CT mediante el Test de Pensamiento Computacional diseñado y verificado por Román-González (2016). obteniendo resultados positivos en la adquisición de conceptos computacionales que establecen la base del CT, tales como la repetición de programas, las direcciones o las funciones simples, lo que ha permitido obtener conclusiones favorables al estudio. Los investigadores Pereira, et al., (2019) presentan una experiencia de enseñar CT a estudiantes de la educación básica a través de actividades de Computación desconectada. Los resultados demuestran el compromiso de los estudiantes y un campo para el trabajo interdisciplinario que involucra CT y disciplinas como las matemáticas.

Por su parte de la Fuente y García (2017), afirman que el CT carece de un marco de referencia común que determine cuál es su definición y los componentes que lo forman y este hecho provoca un vacío en el desarrollo de metodologías y herramientas de evaluación. Los autores proponen valorar el CT de los alumnos de Educación Primaria que han trabajado con Scratch, donde los resultados muestran que el uso de Scratch ayuda al desarrollo de las dimensiones (conceptos, prácticas y perspectivas computacionales) del CT. En el estudio de Palencia (2017), los autores destacan las potencialidades y privilegios pedagógicos que ofrecen las ciencias de la computación para el desarrollo de competencias relacionadas con la resolución creativa de problemas. 
Por su parte, dos Santos, et al. (2019), aplicaron la metodología STEAM para trabajar habilidades de forma más interactiva y autónoma, a través de la producción de Digital Storytelling (DS). Los autores identificaron que las actividades con STEAM y DS ayudaron a los estudiantes a ser más participativos e interesados en su aprendizaje. Del mismo modo, García y García, (2020) realizaron una investigación documental con el objetivo de analizar la metodología STEAM como estrategia de aprendizaje en la asignatura de matemática para estudiantes de bachillerato. Concluyen que se debe redimensionar el currículo educativo, ya que muchos estudiantes que presentan poco interés por el aprendizaje de las matemáticas, debido a su complejidad y profundidad.

\section{Metodología}

El objetivo de este estudio es diagnosticar el nivel de desarrollo en los componentes del CT desde las asignaturas STEAM en la resolución de problemas matemáticos en básica primaria, esto con la finalidad de construir un Test que permita evaluar los componentes del CT en actividades de computación desconectada.

Muestra. En nuestra investigación participaron 22 estudiantes (12 niñas y 10 niños), con un promedio de edad entre los 9 y 10 años. Ellos son estudiantes de grado $4^{\circ}$ de básica primaria, durante el año lectivo 2020.

Enfoque de investigación. De acuerdo a las características de la investigación, esta se realiza bajo el enfoque cualitativo, siguiendo el método de Investigación-Acción. de acuerdo al modelo de Tripp (2005), la Investigación-Acción consiste en un proceso cíclico e iterativo, donde cada ciclo es realizado en cuatro fases: planeación (diagnóstico y planeación de la mejor práctica de acción), acción (poner en práctica la acción planeada), observación (monitorear los efectos de la acción) y evaluación (evaluar y reflexionar sobre los resultados).

La finalidad de la investigación-acción es comprender y resolver problemáticas específicas de una colectividad vinculadas a un ambiente ya sea grupo, programa, organización o comunidad [Hernández y Mendoza, 2018]. Por lo tanto, el alcance de la investigación es de carácter descriptivo.

Procedimiento. Una vez establecida la dificultad para resolución de problemas con división y contando con la muestra de investigación se procede a la elaboración de las consideraciones éticas avaladas por el personal administrativo de la institución educativa y el consentimiento informado de los padres de familia, para seguir con este estudio. Aquí presentamos los dos primeros ciclos de investigación-acción. Cada ciclo se desarrolla en las cuatro fases de Investigación-Acción, descritas a continuación.

\section{Diagnóstico del Pensamiento Computacional para la Resolución de Problemas Matemáticos}

\subsection{Primer Ciclo de Investigación-Acción}

Planeación. Se diseñó un Test para que los estudiantes den respuesta de acuerdo a sus conocimientos previos a situaciones problémicas de división relacionadas con los componentes del CT.

El diseño del Test se realizó en dos etapas. En la primera etapa de preparación se procedió a la busqueda y recolección de problemas matemáticos con división y otros se 
crearon teniendo en cuenta los conocimientos que los estudiantes han adquirido sobre ecosistemas en ciencias naturales y colores secundarios en artística y en la segunda etapa, se adaptaron estos problemas matematicos con división a los componenetes del CT y se organizaron en un cuestionario de Google Docs.

El Test obtenido finalmente consiste en un cuestionario de 20 ítems relacionados con los componentes del CT y modelo STEAM, donde con un ítem se puede medir varios componentes del CT de la siguiente manera: siete (7) ítems del cuestionario permiten determinar si el estudiante es capaz de descomponer el problema en subproblemas más pequeños que al resolverlos ayudaran a encontrar la solución del problema inicial, como el ejemplo mostrado en la Figura 1.

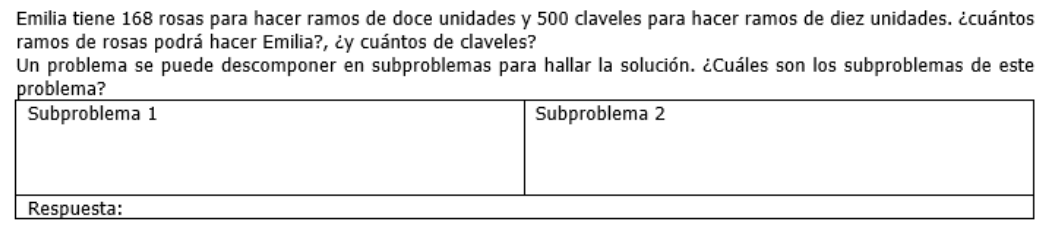

Figura 1. Ejemplo de pregunta de Descomposición.

Cuatro (4) ítems del cuestionario permiten evaluar la abstracción; si el estudiante identifica los datos importantes y que la operación para resolver estos problemas es la división, además sigue en ellos el mismo procedimiento, entonces está generalizando el desarrollo de otros problemas similares (Figura 2).

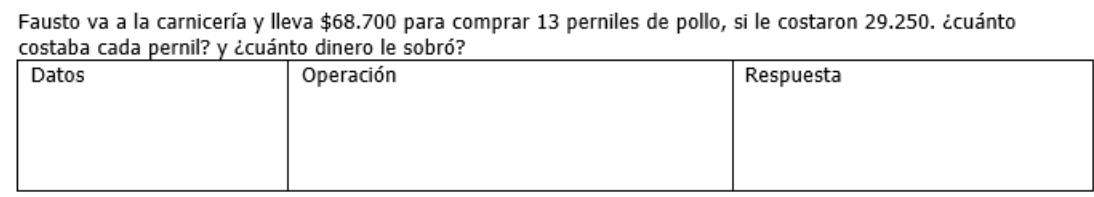

Figura 2. Ejemplo de pregunta de Abstracción-Generalización.

Siete (7) ítems del cuestionario permiten identificar si el estudiante reconoce patrones y el concepto de ciclo pues son problemas que tienen un patrón de desarrollo similar a otros problemas. Cuatro (4) ítems del cuestionario permiten establecer si los estudiantes conocen y practican los pasos de resolución de problemas que están relacionados con la fase de desarrollo de algoritmos (Figura 3). Finalmente, nueve (9) ítems del cuestionario permiten evaluar la Paralelización relacionada con la presencia de la división desde las asignaturas STEAM.

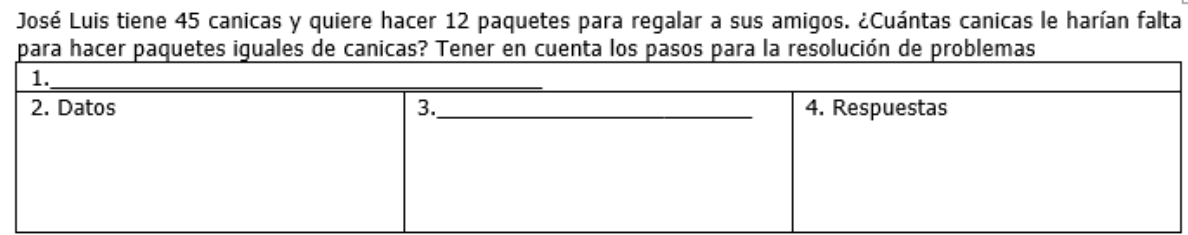

Figura 3. Ejemplo de pregunta de diseño de algoritmos.

Acción. En esta fase se aplica el Test diseñado, entregando a los estudiantes el enlace a través del chat de Google Meet, para que puedan acceder al cuestionario alojado en la plataforma de Google Drive. Para realizar el proceso de recolección de información fueron evaluados los 22 estudiantes en su totalidad. Es así que el Test 
realizado de manera sistemática y pedagógica a través de entornos virtuales se convierte en un instrumento facilitador de información para la toma de decisiones y correctivos.

Observación. Una vez aplicado el cuestionario se revisó la información obtenida en Google Drive y se analizó la codificación que genera Google Docs. Esta información se organiza en una matriz donde se relacionan los componentes del CT con las respuestas dadas por los estudiantes, dando una lectura detallada a cada una de ellas para establecer el nivel de habilidad en el desarrollo de problemas matemáticos aplicando la metodología STEAM y el CT.

Evaluación y Resultados. Evaluamos los resultados obtenidos, y condensamos los datos en un gráfico de barras con los componentes del CT y el porcentaje correspondiente a la cantidad de estudiantes. En la Figura 4, se codifica cada componente sobre el eje horizontal así: Descomposición (D), Abstracción y generalización de patrones (AG), Reconocimiento de patrones-ciclo (RP), Diseño de algoritmos (DA) y Paralelización (P). Sobre el eje vertical están los porcentajes que indican las respuestas dadas por cada estudiante codificada con la palabra No, para indicar que no hace uso de ese componente en la resolución de problemas con división y la palabra $\mathbf{S i}$, para indicar que, si conoce los componentes o por lo menos los practica, lo que permite llegar a conclusiones respecto de los resultados.

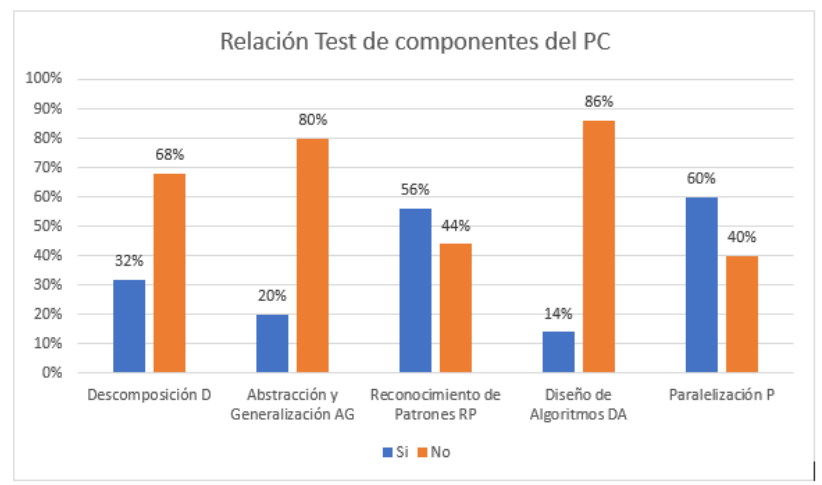

Figura 4. Relación de respuestas del Test de componentes del PC.

A partir de los resultados se puede evidenciar que el No prevalece para los componentes de Descomposición, Abstracción-Generalización y Diseño de Algoritmos. Identificamos que la mayoría de estudiantes realiza la división con ceros al cociente, pero no identifica que el dividendo se descompone para ser repartido en partes iguales entre el divisor; como tampoco comprenden que un problema se puede descomponer en problemas más pequeños para llegar a la solución. Se observa que la mayoría de los estudiantes no identifican cual es la información relevante, no realizan el proceso de abstracción que lleva a la solución de un problema matemático con división y desconocen el diseño de algoritmos en cuanto a los pasos necesarios para la resolución de problemas pues no los aplican en la práctica matemática.

Por otra parte, se identificó que los estudiantes si reconocen o aplican los componentes de Reconocimiento de Patrones y Paralelización. Se evidencia que en su mayoría los estudiantes aplican la paralelización al relacionar la división con otras asignaturas de aprendizaje y reconocen patrones para la resolución de problemas, aunque hay estudiantes que no tienen claro el concepto de ciclo. 
A partir de los resultados, identificamos los componentes del CT que necesitan ser fortalecidos en los estudiantes, no solo desde las matemáticas sino desde otras asignaturas del aprendizaje, es así que optamos por realizar un nuevo ciclo de investigación enfocado en estos aspectos, como se describe a continuación.

\subsection{Segundo Ciclo de Investigación-Acción}

Planeación. En este ciclo buscamos fomentar la habilidad en la resolución de problemas con los componentes del CT a través de actividades de computación desconectada. Para ello, se aplica la metodología STEAM a través del proyecto "Arco Iris" y la interacción con los REDA desde la plataforma Classroom. Esta plataforma permite publicar material educativo para los estudiantes, por su parte el proyecto "Arco Iris" consiste en la elaboración de mini carrozas con temas diversos desde las diferentes asignaturas del STEAM.

Acción. Con la metodología STEAM se llega al conocimiento a través de la construcción donde el estudiante se enfrenta a problemas reales, elaborando mini carrozas en las que se tiene en cuenta la cultura de la región desde las asignaturas de Ciencias Naturales, Tecnología, Artística y Matemáticas, en las que se evidencia la práctica del CT toda vez que los estudiantes expresan a través de sus trabajos la abstracción, descomposición, diseño de algoritmos y paralelización.

En la plataforma Classroom se publicaron diferentes REDA como también vídeos explicativos sobre los componentes del $\mathrm{CT}$, para que los estudiantes practiquen la división y la resolución de problemas matemáticos.

Observación. Una vez terminada la elaboración y montaje de la mini carroza se procede a la observación a través de una lista de cotejo, que permite verificar y evidenciar la práctica del CT a través de los trabajos realizados, así: la abstracción de información en la elaboración de artefactos, la descomposición cuando en familia se dividen tareas de acuerdo a sus habilidades; el desarrollo de algoritmos cuando el estudiante identifica los pasos que siguió para la elaboración de su proyecto y la paralelización cuando explica desde las asignaturas STEAM el algoritmo de la división.

Evaluación y Resultados. La fase de evaluación se realiza con la información recolectada que permite identificar los aprendizajes adquiridos por los estudiantes en las diferentes asignaturas después de la implementación de la metodología STEAM y la interacción con los REDA.

Los resultados muestran que este tipo de actividades incentiva a los estudiantes para la resolución de problemas reales en su contexto social, donde ellos demostraron sus habilidades para relacionar el algoritmo de la división desde diferentes asignaturas del conocimiento. Con la aplicación de esta metodología, los estudiantes desarrollan habilidades en el uso de los componentes de CT siendo los protagonistas en la construcción del conocimiento (figura 5).

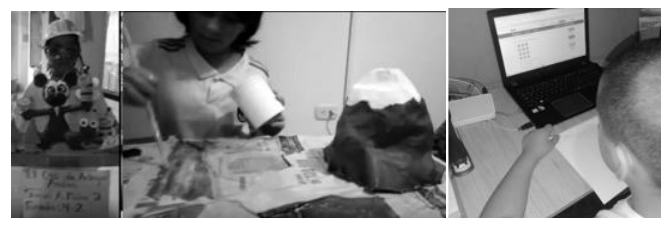

Figura 5. Trabajos realizados por los estudiantes en la elboración de artefactos del proyecto Arco Iris e interacción con los REDA. 
A partir de los resultados aquí obtenidos, identificamos la posibilidad de un siguiente ciclo de Investigación-Acción donde, a partir de la presentación de las mini carrozas a la comunidad educativa de manera virtual, se podrá identificar los aprendizajes y habilidades adquiridas desde otra perspectiva.

\section{Discusión}

La aplicación del Test propuesto ha permitido determinar cuáles son los componentes del CT que presentan más dificultad los estudiantes al momento de resolver un problema matemático, siendo estos la descomposición, abstracción-generalización y diseño de algoritmos, por lo cual se aplica la metodología STEAM a través del Proyecto Arco Iris y la interacción con los REDA. A partir de ellos, identificamos que los estudiantes se interesan por el aprendizaje cuando trabajan poniendo a prueba sus habilidades a través de la construcción de artefactos.

Después de analizar los resultados obtenidos con el test de CT propuesto, se identifica que es necesario modificarlo en cuento al número de preguntas por componente para que la medición de estos sea más equitativa; también se debe hacer cambios en cuanto a la formulación de los problemas con una descripción especifica cuyo desarrollo oriente a medir cada componente del CT. El test corregido será aplicado posteriormente en un nuevo ciclo de Investigación-Acción

Por su parte, el uso de STEAM, como una actividad de computación desconectada, permitió a los estudiantes acercarse al aprendizaje relacionando los conceptos adquiridos con problemas situados en contextos reales donde aplican la ciencia, tecnología, ingeniería, artes y matemáticas; desarrollando la creatividad, habilidad e innovación que permiten estructurar el aprendizaje, la apropiación de los conceptos, la resolución de problema y el desarrollo del pensamiento crítico, importantes para interactuar en la sociedad.

\section{Conclusiones}

El diseño y aplicación del test de pensamiento computacional propuesto permitió determinar en qué componentes del CT los estudiantes presentaron mayor dificultad al momento de resolver problemas matematicos con division, de acuerdo a las dificultades detectadas se desarrolla el proyecto denominado "Arco Iris" con metodología STEAM, la cual permite a través de la creatividad y la construcción poner en practica los componentes del CT.

El desarrollo del proyecto "Arco Iris" con la aplicación de la metodología STEAM a través del cual se contruyen artefactos (mini carrozas), permitieron observar el interes de los estudiantes por el aprendizaje, el cual es mas significativo y del que se apropian con mayor facilidad, logrando potenciar el desarrollo de habilidades del CT. Además se concluye que estas habilidades no hacen parte únicamente de la programación sino que se pueden desarrollar tambien a partir de la implementación de proyectos interdisciplinarios que propicien en su construcción el desarrollo del CT. 
La interacción con los recursos educativos digitales REDA a través de la plataforma classroom, permitió potenciar el nivel de desarrollo en la resolución de problemas con CT. La evaluación de las actividades interactiva con estos recursos, se realizará posteriormente por encontrarse en proceso de implementación.

A partir del estudio realizado, identificamos los beneificios de la aplicación del test de CT, el uso de la metodología STEAM por medio de proyectos y la aplicación de los REDA. El Test permitió un diagnóstico inicial de las habilidades de los componentes del $\mathrm{CT}$ en los estudiantes para diseñar actividades acordes a ese diagnóstico. Posteriormente, el proyecto realizado con STEAM y los REDA aplicando pensamiento computacional, incentivaron a los estudiantes al resolver problemas matemáticos con división, donde no se limitaron a responder la pregunta del problema sino que fueron más allá, aplicando la descomposición, abstracción, reconocimiento de patrones, diseño de algoritmos y paralelización como componentes de CT.

Los investigadores proponen en el tema de resolución de problemas matemáticos con pensamiento computacional, la aplicación de un test para determinar el nivel de desarrollo de sus componentes, una vez determinadas las dificultades, la aplicación de una metodología que contribuya al desarrollo de estos componentes como es el caso de la metodogia STEAM a través del desarrollo de proyectos y el uso de los REDA.

\section{Referencias}

Bell, T., Alexander, J., Freeman, I., \& Grimley, M. (2009). Computer science unplugged: School students doing real computing without computers. The New Zealand Journal of Applied Computing and Information Technology, 13(1), 20-29.

Berrocoso, J. V., Sánchez, M. R. F., \& Arroyo, M. D. C. G. (2015). El pensamiento computacional y las nuevas ecologías del aprendizaje. Revista de educación a distancia, (46).

Brennan, K., \& Resnick, M. (2012, April). New frameworks for studying and assessing the development of computational thinking. In Proceedings of the 2012 annual meeting of the American educational research association, Vancouver, Canada (Vol. 1, p. 25).

de la Fuente, H. A., \& García, A. P. (2017). Evaluación del Pensamiento Computacional en Educación Primaria. Revista Interuniversitaria de Investigación en Tecnología Educativa. Educativa (RIITE), No 3, pp. 25-39.

Doleck, T., Bazelais, P., Lemay, D. J., Saxena, A., \& Basnet, R. B. (2017). Algorithmic thinking, cooperativity, creativity, critical thinking, and problem solving: exploring the relationship between computational thinking skills and academic performance. Journal of Computers in Education, 4(4), 355-369.

dos Santos Silva, D. E., Sobrinho, M. C., \& Valentim, N. (2019, November). STEAM and Digital Storytelling: a case study with high school students in the context of Education 4.0. In Brazilian Symposium on Computers in Education (Simpósio Brasileiro de Informática na Educação-SBIE) (Vol. 30, No. 1, p. 159).

García-Mejía, R. O., \& García-Vera, C. E. (2020). Metodología STEAM y su uso en Matemáticas para estudiantes de bachillerato en tiempos de pandemia Covid19. Dominio de las Ciencias, 6(2), 163-180. 
IX Congresso Brasileiro de Informática na Educação (CBIE 2020)

Anais do XXVI Workshop de Informática na Escola (WIE 2020)

Hernández, R. , Mendoza, C. (2018). Metodología de la investigación. McGraw-Hill Interamericana. Tomado de https://www.ebooks7-24.com:443/?il=6443

Oliveira, C. M., Pereira, R., Galvão, L., Peres, L., \& Schultz, E. (2019, November). Utilização de Desafios para o Desenvolvimento do Pensamento Computacional no Ensino Superior: um relato de experiência. In Brazilian Symposium on Computers in Education (Simpósio Brasileiro de Informática na Educação-SBIE) (Vol. 30, No. 1, p. 2005).

Ortega, J., Guerrero, J., \& Romo, J. L. (2016). Desarrollo del pensamiento computacional a través de juegos y actividades lúdicas sin uso del computador. Tesis Universidad de Nariño-Colombia.

Palencia, M. P. (2017). El pensamiento computacional y la resolución de problemas: una apuesta pedagógica en el siglo XXI. Revista Boletín Redipe, 6(8), 63-73.

Pereira, F. T. S. S., Araújo, L. G., \& Bittencourt, R. (2019, November). Intervenções de Pensamento Computacional na Educação Básica através de Computação Desplugada. In Anais do Workshop de Informática na Escola (Vol. 25, No. 1, p. 315).

Pérez Narváez, H. O., Álvarez-Zurita, A., \& Guevara Herrera, C. R. (2019). Dominio de habilidades del pensamiento computacional en los estudiantes del Instituto Tecnológico Superior Sucre de Quito - Ecuador. Revista Interuniversitaria De Investigación En Tecnología Educativa, (7), 48-60.

Rodríguez, M. Á. (2017). Desarrollo del pensamiento computacional en educación primaria: una experiencia educativa con Scratch. Universitas Tarraconensis. Revista de Ciències de l'Educació, 1(2), 45-64.

Román-González, M., Pérez-González, J. C., \& Jiménez-Fernández, C. (2015, October). Test de Pensamiento Computacional: diseño y psicometría general. In III Congreso Internacional sobre Aprendizaje, Innovación y Competitividad (CINAIC 2015) (pp. $1-6)$.

Román-González, M. (2016). Código alfabetización y pensamiento computacional en education primaria y secundaria: Validación de un instrumento y evaluación de programas (tesis doctoral). Universidad Nacional de Educación a Distancia. Escuela Internacional de Doctorado. España

Shute, V. J., Sun, C., \& Asbell-Clarke, J. (2017). Demystifying computational thinking. Educational Research Review, 22, 142-158.

Tripp, D. (2005). Pesquisa-ação: uma introdução metodológica. Educação $e$ pesquisa, 31(3), 443-466.

Weintrop, D., Beheshti, E., Horn, M., Orton, K., Jona, K., Trouille, L., \& Wilensky, U. (2016). Defining computational thinking for mathematics and science classrooms. Journal of Science Education and Technology, 25(1), 127-147.

Wing, J. M. (2008). Computational thinking and thinking about computing. Philosophical Transactions of the Royal Society A: Mathematical, Physical and Engineering Sciences, 366(1881), 3717-3725.

Yadav, A., Hong, H., \& Stephenson, C. (2016). Computational thinking for all: pedagogical approaches to embedding 21 st century problem solving in K-12 classrooms. TechTrends, 60(6), 565-568. 